\title{
Fatal pulmonary embolism caused by thrombosis of contralateral axillary vein after arthroscopic right rotator cuff repair
} -A case report-

\author{
Seok Jai Kim, Kyung Yeon Yoo, Hyung-Gon Lee, Woong-Mo Kim, Cheol Won Jeong, and Hyun-Jung Lee \\ Department Anesthesiology and Pain Medicine, Chonnam National University Medical School, Gwangju, Korea
}

A clinically apparent thromboembolism associated with arthroscopic shoulder surgery is extremely rare. We report a case of a fatal pulmonary embolism developed after an arthroscopic rotator cuff repair in a 45-year-old woman. On the first day after surgery, she experienced syncope that was complicated by cardiac arrest. No hemostasis impairment was noted. A computed tomography scan revealed a pulmonary embolism, and Doppler ultrasound revealed thrombosis of the axillary vein on the contralateral shoulder. She died from multiple organ failure 13 days after surgery. This case shows that clinicians must be aware of the potential occurrence of a pulmonary thromboembolism in patients undergoing prolonged arthroscopic shoulder surgery. (Korean J Anesthesiol 2010; 59: S172-S175)

Key Words: Deep venous thrombosis, Fatal pulmonary embolism, Rotator cuff repair, Shoulder arthroscopy.

Deep venous thrombosis (DVT) is a common and often lifethreatening complication of orthopedic surgery, particularly hip and knee surgery [1]. Shoulder arthroscopy is being used increasingly to perform a variety of diagnostic and therapeutic procedures around the shoulder. Although the incidence of thromboembolic complications after this type of surgery is low [2], major complications associated with upper extremity DVT (UEDVT), including pulmonary embolism (PE) and death, have been reported [2-7]. However, there are no reports of $\mathrm{PE}$ associated with a thrombosis of the contralateral upper extremity venous system after arthroscopic shoulder surgery.
We report a case of 45-year-old woman who developed thrombosis of the contralateral axillary vein complicated by a fatal PE after an arthroscopic right rotator cuff repair.

\section{Case Report}

A 45-year-old woman (weight, $74 \mathrm{~kg}$; height, $163 \mathrm{~cm}$; body mass index, $27.9 \mathrm{~kg} / \mathrm{m}^{2}$ ) presented for arthroscopic rotator cuff repair due to rotator cuff tear. She had a 5-year-history of type 2 diabetes mellitus, which was treated with oral hypoglycemic agents. She received no other medications. The patient denied

Received: March 5, 2010. Revised: 1st, March 20, 2010; 2nd, March 30, 2010. Accepted: April 20, 2010.

Corresponding author: Kyung Yeon Yoo, M.D., Ph.D., Department Anesthesiology and Pain Medicine, Chonnam National University Medical School, 8, Hak 1-dong, Dong-gu, Gwangju 501-757, Korea. Tel: 82-62-220-6893, Fax: 82-62-232-6294, E-mail: kyyoo@jnu.ac.kr

(c) This is an open-access article distributed under the terms of the Creative Commons Attribution Non-Commercial License (http:// creativecommons.org/licenses/by-nc/3.0/), which permits unrestricted non-commercial use, distribution, and reproduction in any medium, provided the original work is properly cited. 
any symptoms of deep vein thrombosis or other vascular disease. In addition, she had no smoking history. The routine laboratory data, electrocardiogram and chest radiograph revealed no serious abnormalities.

She was premedicated with midazolam ( $3.75 \mathrm{mg}$, orally) 60 min before induction of anesthesia. General anesthesia was induced with intravenous propofol $(2 \mathrm{mg} / \mathrm{kg}$ ), lidocaine (40 $\mathrm{mg}$ ) and rocuronium $(0.9 \mathrm{mg} / \mathrm{kg})$. Intubation was performed when the neuromuscular block had been achieved, and anesthesia was maintained with sevoflurane $1.5 \%$ and a continuous infusion of remifentanil at $0.1-0.5 \mu \mathrm{g} / \mathrm{kg} / \mathrm{min}$. The patient was ventilated mechanically using a ventilator to maintain end-tidal carbon dioxide (CO2) tension between $35-40 \mathrm{mmHg}$. The bispectral index value was maintained at 40-50. The neuromuscular blockade was controlled carefully during the surgical procedure by train-of-four monitoring. The end-tidal oxygen, $\mathrm{CO}_{2}$, and sevoflurane levels were measured continuously using an infrared analyzer (Datax, Model 254, Helsinki, Finland).

When general anesthesia was induced successfully, the arthroscopic rotator cuff repair on the right shoulder was performed in the beach chair position. Surgery lasted $6 \mathrm{~h}$ and was uneventful. The patient was taken to the recovery room in a stable condition. The initial postoperative recovery was uneventful, and the patient was transferred to the ward.

The next morning at $7: 00$, the patient was found to have stable vital signs and without complaints. At $10: 10$, she became lightheaded and knelt down on the floor on her way to the

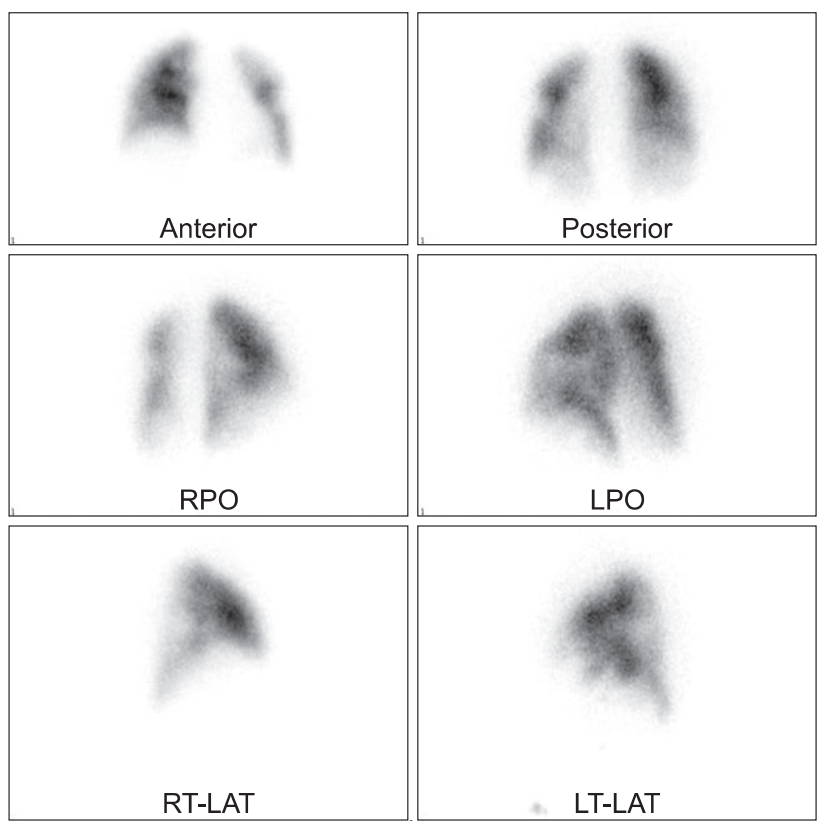

Fig. 1. Tc-99m MAA lung perfusion scan shows no significant perfusion defect. bathroom. She was minimally responsive to commands in respiratory distress and showed a thready pulse. A few minutes later, she developed cardiac arrest. The emergency code team was called. Full cardiopulmonary resuscitation (CPR) was started immediately with defibrillation and epinephrine. Spontaneous circulation was resumed 60 minutes later. Her systolic blood pressure (BP) was $80 \mathrm{mmHg}$ and heart rate (HR) was approximately 100 beats/min under the continuous infusion of dopamine $10 \mu \mathrm{g} / \mathrm{kg} / \mathrm{min}$ and norepinephrine $0.5 \mu \mathrm{g} / \mathrm{kg} / \mathrm{min}$. Under an inspired oxygen fraction of 0.4 , the arterial blood gas analysis revealed the following: $\mathrm{pH} 7.33$, $\mathrm{PaCO}_{2} 45 \mathrm{mmHg}, \mathrm{PaO}_{2} 80 \mathrm{mmHg}, \mathrm{HCO}_{3}{ }^{-} 23.7 \mathrm{mM} / \mathrm{L}$, and $\mathrm{SaO}_{2}$ 99\%. Emergent transthoracic echocardiography was performed to determine if a PE was the cause of the cardiovascular collapse, and revealed a dilated right ventricle, a leftward septal bowing, tricuspid regurgitation, and decreased left ventricular chamber size without a definite configuration of the D-shape of the left ventricle, which is compatible with a diagnosis of PE (not shown). The electrocardiogram was within the normal range, and the chest radiograph showed similar findings as the one before surgery. Based on the echocardiography findings of a suspicious PE and an increased D-dimer level of $8.58 \mathrm{mg} / \mathrm{L}$ (normal range, $0-0.3 \mathrm{mg} / \mathrm{L}$ ), $1 \mathrm{mg} / \mathrm{kg}$ Enoxaparin (Clexane $^{\circledR}$, low-molecular weight heparin) was administered subcutaneously, twice daily as recommanded [8]. Lung perfusion scintigraphy taken on the 3rd postoperative day revealed no significant abnormalities (Fig. 1). However, the pulmonary angiographic scan showed emboli in the right and left lower lobar pulmonary arteries (Fig. 2).

On the 5th postoperative day, a Doppler ultrasound scan

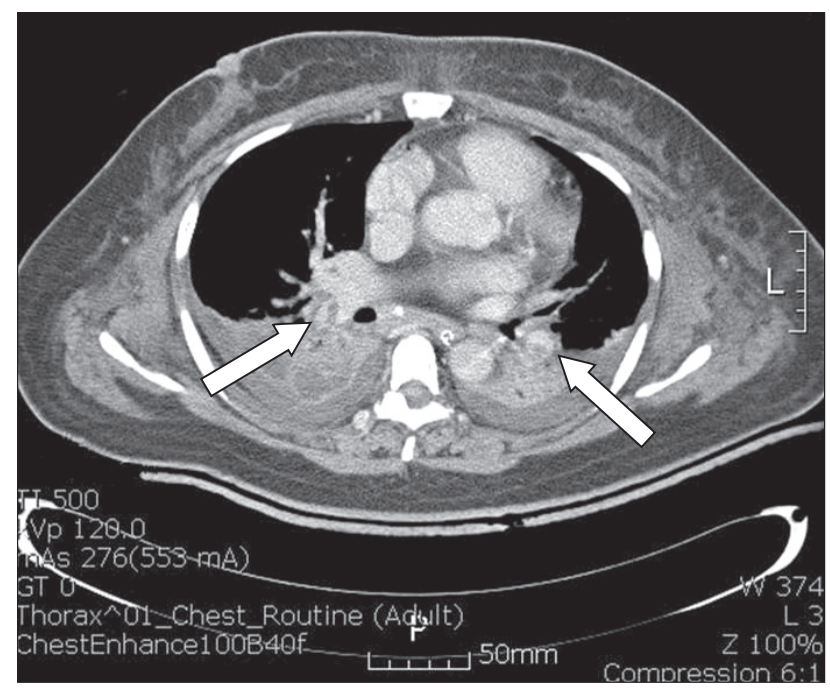

Fig. 2. Computed tomography pulmonary angiogram shows emboli (intraluminal filling defect) in the right lower lobar, and left lower lobar pulmonary arteries (arrow). 


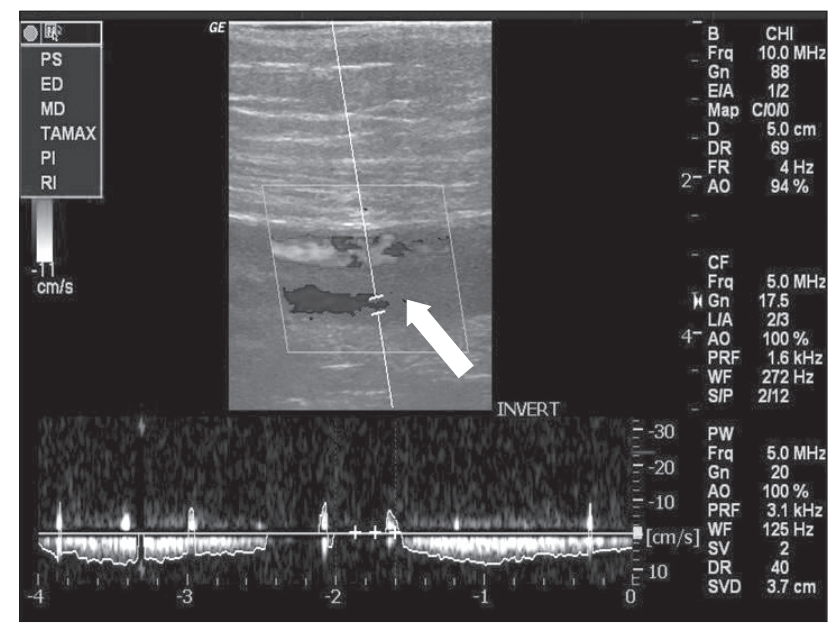

Fig. 3. Extremity venous Doppler ultrasound shows a non-collapsible, solid left axillary vein (arrow).

revealed a non-collapsible, solid axillary vein on the contralateral side compared to a collapsible vein on the right (Fig. 3). The findings of the lower limbs and iliac vein were normal. Under a low dose dopamine infusion, the systolic BP was 120$140 \mathrm{mmHg}$ and HR $80-100$ beats/min. However, the patient was comatose and anuric from admission to the intensive care unit and deteriorated progressively. The electroencephalogram and brain computed tomography (CT) confirmed a hypoxic cerebral dysfunction. She died of multiple organ failure on the 13th postoperative day.

\section{Discussion}

The development of UEDVT has been associated with anatomical abnormalities causing venous compression, strenuous upper extremity exercise, venous catheterization as with pacemakers, venous irritation or compression by the shaver, and subcutaneous edema around shoulder by the extravasation of irrigation fluid, excessive arm traction, and inadequate positioning of the arm [3]. Two cases of UEDVT after shoulder arthroscopy were associated with a lateral decubitus position $[2,9]$, and another two cases were associated with a beach chair position $[3,4]$. In addition, postoperative thromboembolic diseases are related to several risk factors, including diabetes and obesity [5]. In one case report, diabetic and obese subjects undergoing shoulder arthroscopic surgery presented with severe, life-threatening UEDVT and PE despite the immediate postoperative low-dose heparin prophylaxis [7]. Long surgical procedures may affect the clotting cascade or result in the release of thrombogenic factors [10]. Overall, diabetes and obesity, as the patient's risk factors, and prolonged surgery in the beach chair position may have contributed to the UEDVT and subsequent PE in our case.
The pulmonary emboli that develop after upper extremity surgery arise mainly from the ipsilateral axillary subclavian venous system [6] or either of the lower extremities [6]. Ipsilateral venous injuries have been associated with venous irritation or compression by the shaver, subcutaneous edema around the shoulder by the extravasation of irrigation fluid, excessive arm traction, and inadequate positioning of the arm [3]. In our case, in contrast to many reports, the thrombosis was on the contralateral axillary vein.

The symptoms of UEDVT may vary from asymptomatic to pain and/or swelling of the arm, a prominence of superficial veins in the upper arm and chest, cyanosis of the hand, numbness and tingling of the fingers and functional impairment [11]. A PE with UEDVT must be ruled out if shortness of breath, pleuritic chest pain or other symptoms of pulmonary distress are noted, even though most pulmonary emboli are not clinically obvious [12]. The symptoms and signs of PE usually develop several days after surgery [2]. However, in our case, a syncopal episode and subsequent cardiac arrest developed within 15 hours after surgery, highlighting the importance of early diagnosis and treatment of thromboembolism or it prevention.

Objective tests diagnosing PE are very important because a clinical assessment alone is unreliable and may cause a misdiagnosis. There are many valuable tests including spiral CT, ventilation-perfusion lung scan, pulmonary artery angiography and echocardiography (both transthoracic and transesophageal). A CT angiogram is considered a criterion standard for a diagnosis of PE but no single test for PE is both sensitive and specific [13]. In particular, echocardiography is feasible, low risk and inexpensive in critically ill patients, in whom other diagnostic techniques are highly risky or technically difficult. Furthermore, echocardiography does not interfere with resuscitation efforts. Therefore, it is the method of choice for substantiating a diagnosis of PE in patients ongoing CPR. Our patient showed PE in the pulmonary CT angiogram, even though the findings of the lung scan were negative.

Different therapeutic strategies, including thrombolytic therapy, surgical embolectomy, and anticoagulation in patients with a cardiac arrest caused by PE have been used to reduce the mortality. Thrombolysis is the first line treatment for massive PE and may be instituted on clinical grounds alone if cardiac arrest is imminent. One advantage of their use is that thrombolytic agents, such as urokinase, streptokinase and rt-PA are easily and rapidly available in almost all hospitals. However, bleeding, in particular cerebral bleeding with a fatal or disabling outcome is the main hazard of thrombolytic therapy. In our case, thrombolytic agents were not administered due to the concerning for bleeding at the site of surgery and spontaneous circulation was returned before their use was considered. Surgical embolectomy for the treatment of cardiac arrest caused 
by PE is useful as an alternative method, particularly after failure of thrombolysis or when this is contraindicated, e.g. previous major trauma and/or recent surgery [14]. Anticoagulation has become the minimum appropriate intervention for UEDVT management. In massive PE, unfractionated heparin should be considered as a first dose bolus. Anticoagulation has been reported to prevent clot propagation, to facilitate the maintenance of venous collaterals and prevent PE $[8,15]$. For all patients undergoing shoulder surgery, early ambulation, perioperative thigh-high elastic stockings, thigh-high pneumatic compressive devices, and occasionally pharmacologic prophylaxis, including low molecular weight heparin, factor Xa inhibitor, adjusted-dose vitamin $\mathrm{K}$ antagonist and aspirin, are recommended [8].

In conclusion, this case highlights the possibility of a potentially fatal perioperative pulmonary thromboembolism occurring after arthroscopic shoulder surgery and emphasizes the importance of early diagnosis and treatment or prevention to limit the morbidity and mortality.

\section{References}

1. Demers C, Marcoux S, Ginsberg JS, Laroche F, Cloutier R, Poulin J. Incidence of venographically proved deep vein thrombosis after knee arthroscopy. Arch Intern Med 1998; 158: 47-50.

2. Polzhofer GK, Petersen W, Hassenpflug J. Thromboembolic complication after arthroscopic shoulder surgery. Arthroscopy 2003; 19: E129-32.

3. Cortés ZE, Hammerman SM, Gartsman GM. Pulmonary embolism after shoulder arthroscopy: could patient positioning and traction make a difference? J Shoulder Elbow Surg 2007; 16: e16-7.

4. Creighton RA, Cole BJ. Upper extremity deep venous thrombosis after shoulder arthroscopy: a case report. J Shoulder Elbow Surg 2007; 16: e20-2.

5. Rockwood CA Jr, Wirth MA, Blair S. Warning: pulmonary embolism can occur after elective shoulder surgery-report of two cases and survey of the members of the American Shoulder and Elbow Surgeons. J Shoulder Elbow Surg 2003; 12: 628-30.

6. Willis AA, Warren RF, Craig EV, Adler RS, Cordasco FA, Lyman S, et al. Deep vein thrombosis after reconstructive shoulder arthroplasty: a prospective observational study. J Shoulder Elbow Surg 2009; 18: $100-6$.

7. Polzhofer GK, Petersen W, Hassenpflug J. Thromboembolic complication after arthroscopic shoulder surgery. Arthroscopy 2003; 19: E129-32.

8. Muntz JE, Michota FA. Prevention and management of venous thromboembolism in the surgical patient: options by surgery type and individual patient risk factors. Am J Surg 2010; 199: S11-20.

9. Burkhart SS. Deep venous thrombosis after shoulder arthroscopy. Arthroscopy 1990; 6: 61-3.

10. Mansfield AO. Alteration in fibrinolysis associated with surgery and venous thrombosis. Br J Surg 1972; 59: 754-7.

11. Prandoni P, Bernardi E. Upper extremity deep vein thrombosis. Curr Opin Pulm Med 1999; 5: 222-6.

12. Ascher E, Salles-Cunha S, Hingorani A. Morbidity and mortality associated with internal jugular vein thromboses. Vasc Endovascular Surg 2005; 39: 335-9.

13. Kearon C. Diagnosis of pulmonary embolism. CMAJ 2003; 168: 18394.

14. Ullmann M, Hemmer W, Hannekum A. The urgent pulmonary embolectomy: mechanical resuscitation in the operating theatre determines the outcome. Thorac Cardiovasc Surg 1999; 47: 5-8.

15. Hingorani A, Ascher E, Lorenson E, DePippo P, Salles-Cunha S, Scheinman M, et al. Upper extremity deep venous thrombosis and its impact on morbidity and mortality rates in a hospital-based population. J Vasc Surg 1997; 26: 853-60. 